\title{
Compressed sensing in MRI - mathematical preliminaries and basic examples
}

\author{
Eukasz Błaszczyk
}

\begin{abstract}
In magnetic resonance imaging (MRI), $k$-space sampling, due to physical restrictions, is very time-consuming. It cannot be much improved using classical Nyquist-based sampling theory. Recent developments utilize the fact that MR images are sparse in some representations (i.e. wavelet coefficients). This new theory, created by Candès and Romberg, called compressed sensing (CS), shows that images with sparse representations can be recovered from randomly undersampled $k$-space data, by using nonlinear reconstruction algorithms (i.e. $l_{1}$-norm minimization). Throughout this paper, mathematical preliminaries of CS are outlined, in the form introduced by Candès. We describe the main conditions for measurement matrices and recovery algorithms and present a basic example, showing that while the method really works (reducing the time of MR examination), there are some major problems that need to be taken into consideration.
\end{abstract}

Key words: compressed sensing $\bullet$ magnetic resonance imaging $\bullet$ sampling theory $\bullet$ sparsity

\section{Ł. Błaszczyk}

Institute of Radioelectronics,

Warsaw University of Technology,

15/19 Nowowiejska Str., 00-665 Warsaw, Poland,

Tel.: +48 22234 7647,

E-mail: L.Blaszczyk@ire.pw.edu.pl

Received: 2 July 2014

Accepted: 5 August 2015

\section{Introduction}

Data acquired in magnetic resonance imaging (MRI) is a function $X: \mathbf{R}^{3} \rightarrow \mathbf{C}$ describing magnetic properties of the matter in space. Protons are of main interest, characterized by their magnetic moment, processing in the constant magnetic field $B_{0}$ with Larmor frequency $\omega=-\gamma B_{0}$, where $\gamma$ is a gyromagnetic ratio. Obtaining the signal from many protons requires the differentiation of their precession frequencies. To do so, gradient magnetic fields $\mathbf{G}$ : $[0, T] \rightarrow \mathbf{R}^{3}$ are applied, defining $k$-space sampling trajectories:

$$
\mathbf{k}(t)=\frac{\gamma}{2 \pi} \int_{0}^{t} \mathbf{G}(\tau) d \tau
$$

Acquired signal is the Fourier transform of the magnitude of MR image

$$
f(t)=\int_{\mathbf{R}^{3}}|X(\mathbf{z})| \cdot \exp \{-2 \pi j\langle\mathbf{k}(t), \mathbf{z}\rangle\} d \mathbf{z}=F\{|X|\}(\mathbf{k}(t))
$$

sampled along the trajectories $\mathbf{k}(t)$, as shown in Fig. 1 [1]. Due to physical restrictions, we need more than one trajectory to sample the whole $k$-space. Discretization is required and in consequence Nyquist sampling rate must be satisfied. It results in long signal acquisition and low image resolution. At the same time, the data is compressible, which allows us to utilize a recently developed technique, called compressed sensing (CS).

Image acquisition process in MRI, based on Fourier transform, is linear. The task is then to 

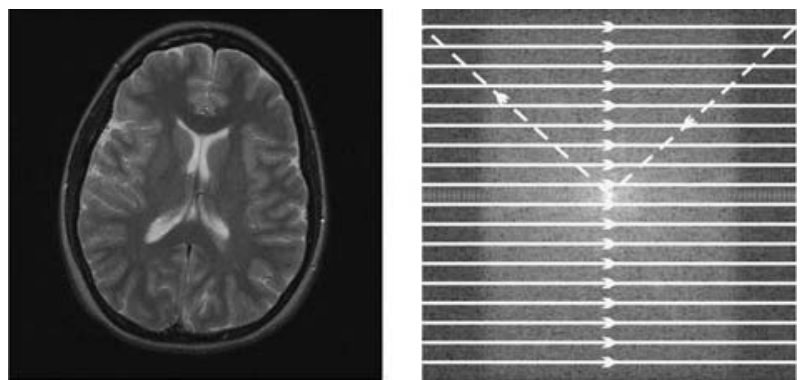

Fig. 1. A classic example of MR image (T2-weighted brain image) and $k$-space sampling pattern. On the left: MR image example $(512 \times 512 \mathrm{px})$, source [2]. On the right: scheme of $N$ Fourier lines $k$-space sampling pattern, lines and arrows show the direction of $k$-space acquisition.

solve the system of linear equations $\Phi \mathbf{x}=\mathbf{y}$, where $\mathbf{x} \in \mathbf{C}^{n}$ is MR signal, $y \in \mathbf{C}^{m}$ is a vector of samples and $\Phi \in \mathbf{C}^{m \cdot n}$ is a measurement matrix [2]. When $m<n$, such a system is underdetermined, which means that if there exists at least one solution, then the system has infinitely many solutions. To have a single unique solution, we require $m$ to satisfy $m \geq n$. On the other hand, we would like $m$ to be lower than the number of samples required by Nyquist rate, $n$. Compressed sensing theory provides stable algorithms that allow us to uniquely reconstruct $\mathbf{x} \in \mathbf{C}^{n}$ from $m<<n$ samples for some special classes of signals, called sparse signals.

\section{Compressed sensing - mathematical preliminaries}

Compressed sensing theory is based on sparsity of the signal, as was shown in [2].

Definition 1. Let $\|\mathbf{x}\|_{0}$ denote a number of non-zero elements of a vector $\mathbf{x}$. Signal $\mathbf{x} \in \mathbf{C}^{n}$ is called $s$-sparse if $\|\mathbf{x}\|_{0} \leq s$.

Theorem 1. Signal $\|\mathbf{x}\|_{0}$ is a unique $s$-sparse solution to the system $\mathbf{y}=\Phi \mathbf{z}$ if and only if it is a unique solution to the $l_{0}$-minimization problem

$$
\underset{\mathbf{z} \in \mathbf{C}^{n}}{\arg \min }\|\mathbf{z}\|_{0} \text { with } \Phi \mathbf{z}=\mathbf{y}
$$

Most of the real-life signals are sparse or compressible ('nearly sparse') in their original base or in some representation (i.e. wavelet transform). So, if it is possible to discard most of the data without any loss of information, then it is unnecessary to acquire so much data. It is visible in magnetic resonance imaging (MRI), which is both time-consuming and compressible (Fig. 2).

Unfortunately, $l_{0}$-minimization problem is NP-hard. Consider a convex minimization problem (called $l_{1}$-minimization):

$$
\underset{\mathbf{z} \in \mathbf{C}^{n}}{\arg \min }\|\mathbf{z}\|_{1} \text { with } \Phi \mathbf{z}=\mathbf{y}
$$

There are certain conditions under which both problems are equivalent, known under the name null space property [3].

Definition 2. We say that $\Phi \in \mathbf{C}^{m \cdot n}$ satisfies null space property (NSP) of order $s$ if $\left\|\mathbf{x}_{s}\right\|_{0}<\left\|\mathbf{x}_{S} c\right\|_{1}$ for all subsets $S \subseteq\{1, \ldots, n\}, \# S \leq s$, and all vectors $\mathbf{x} \in \operatorname{ker} \Phi \backslash\{0\}$, where $S^{c}=\{1, \ldots, n\} \backslash S$.
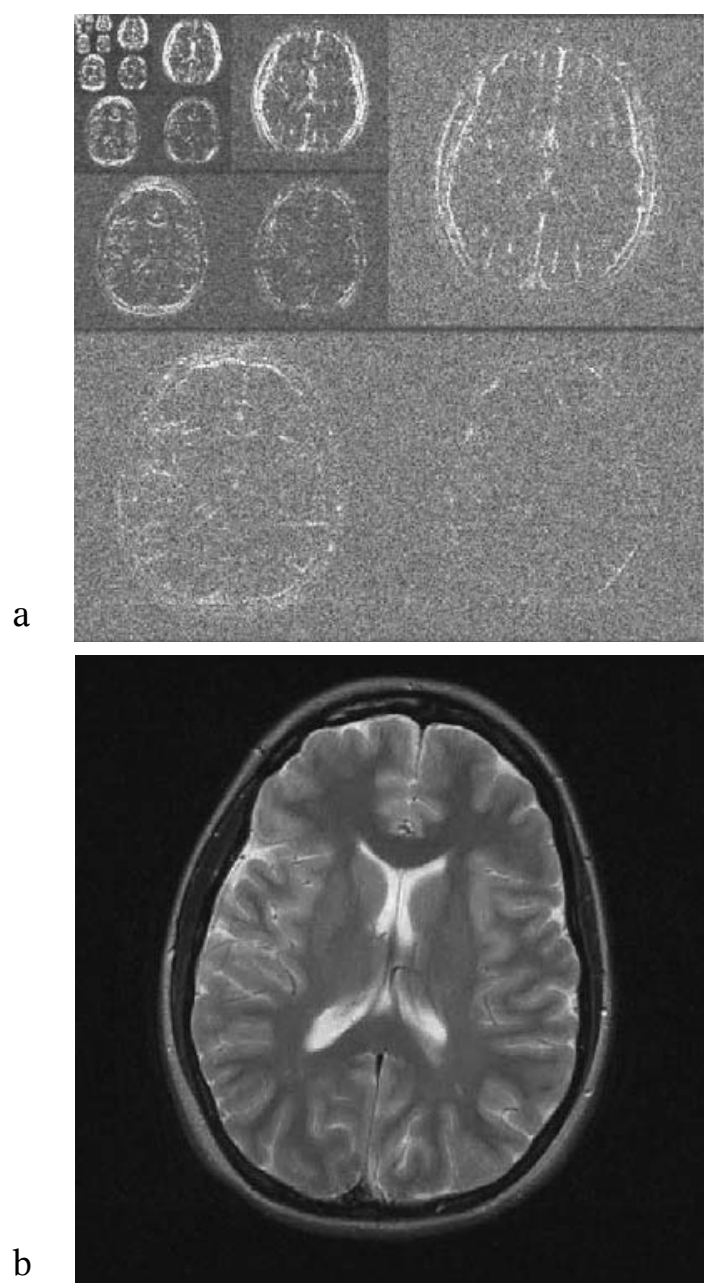

Fig. 2. An example of MR image compressibility. Popular Daubechies wavelets were applied to compress the image and the reconstructed was obtained from $10 \%$ of wavelet transform largest coefficients, showing almost no loss of information. (a) Wavelet transform of the image (from Fig. 1). (b) Image reconstructed from 10\% of wavelet transform largest coefficients.

Theorem 2. Let $\Phi \in \mathbf{C}^{m \cdot n}$ denote a measurement matrix. Any $s$-sparse vector $\mathbf{x} \in \mathbf{C}^{n}$ is a unique solution to the problem (4) if and only if $\Phi$ satisfies NSP of order $s$.

For such $\Phi$, the solution of (4) solves also the problem (3). While it is computationally complex to show that matrix satisfies NSP, there is another property that allows to find a solution to the regarded problem. It is called restricted isometry property [4].

Definition 3. The $s$-th restricted isometry constant $\delta_{s}$ of $\Phi \in \mathbf{C}^{m \cdot n}$ is the smallest $\delta_{s} \geq 0$ such that for all $s$-sparse vectors $\mathbf{x} \in \mathbf{C}^{n}$

$$
\left(1-\delta_{s}\right)\|\mathbf{x}\|_{2}^{2} \leq\|\Phi \mathbf{x}\|_{2}^{2} \leq\left(1+\delta_{s}\right)\|\mathbf{x}\|_{2}^{2}
$$

We say that $\Phi$ satisfies restricted isometry property (RIP) if $\delta_{s}$ is small for reasonably large $s$.

Theorem 3. Let $\delta_{2}<\sqrt{2}-1$. If $\mathbf{x}^{\#}$ is the solution to (4) then

$$
\begin{aligned}
& \left\|\mathbf{x}^{\#}-\mathbf{x}\right\|_{2} \leq \frac{C_{0}}{\sqrt{s}}\left\|\mathbf{x}-\mathbf{x}_{s}\right\|_{1} \text { and } \\
& \left\|\mathbf{x}^{\#}-\mathbf{x}\right\|_{1} \leq C_{0}\left\|\mathbf{x}-\mathbf{x}_{s}\right\|_{1}
\end{aligned}
$$



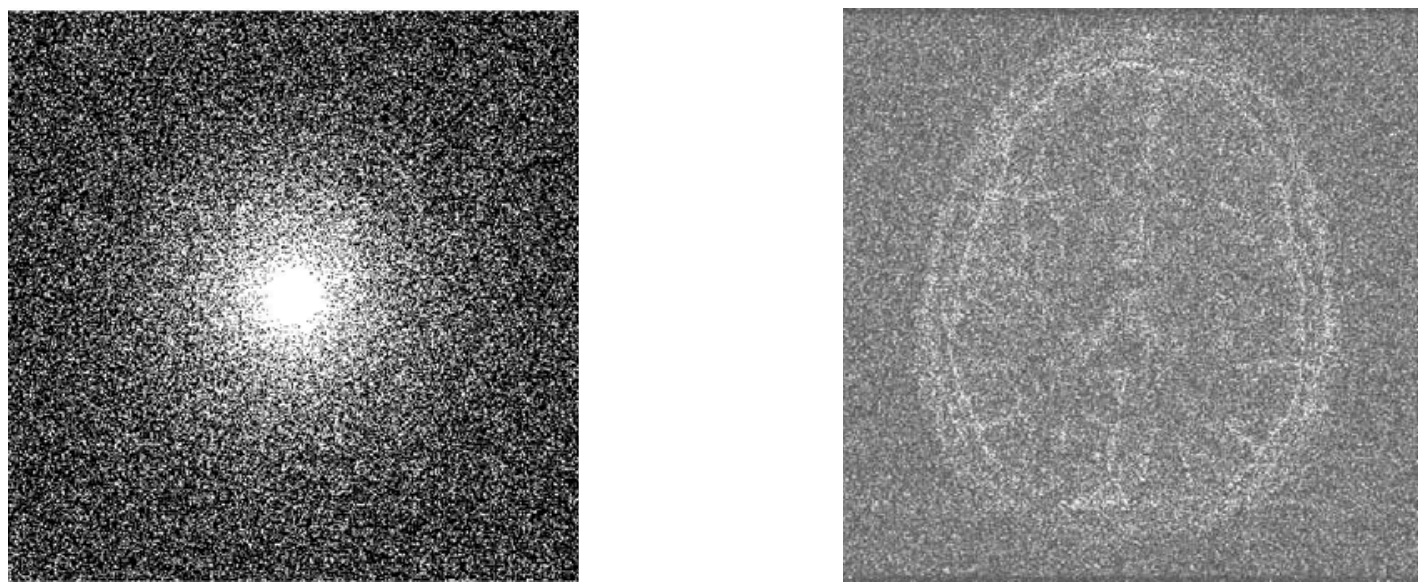

Fig. 3. $k$-Space sampling mask, and map of the absolute error of CS reconstruction from randomly sampled $k$-space data.

where $\mathbf{x}_{s}$ is the best $s$-sparse approximation of $\mathbf{x}$ and constant $C_{0}$ depends on $\delta_{2 s}$.

The above result, proved in [4], means that if $\mathbf{x}$ is $s$-sparse then the reconstruction is exact.

\section{Compressed sensing in MRI - basic example}

The basic example of a 'good' measurement matrix is the Fourier transform matrix [5].

Theorem 4. If $n$ is a prime number then any $s$-sparse signal $\mathbf{x} \in \mathbf{C}^{n}$ can be uniquely reconstructed from $m \geq 2 s$ uniformly chosen Fourier coefficients $\widehat{\mathbf{x}}$.

In the real-life applications, it is harder than that. Measurements are susceptible to errors and the signal is not sparse but compressible (and usually not in the original base but in some representation, i.e. wavelet transform). Nevertheless this method works (with minor changes), because not all of the assumptions are strictly required (i.e. asymptotic incoherence).

Given that MR images are compressible in wavelet domain and data acquisition is based on Fourier transform, it is natural to utilize compressed sensing methods, such as described above. Some modifications need to be made (such as non-uniform random sampling), but basics of the method still hold (Fig. 3)

\section{Remarks}

In MR tomography, it is not possible to sample $k$-space completely at random, but it is possible to choose random parallel $k$-space trajectories. While in
2D case it means randomness only in one dimension, better results are obtained when we are dealing with 3D data acquisition (maybe 4D acquisition, which includes time, would be even better because of the randomness in three dimensions). Unfortunately, at this point studies are mainly academic; however, one of the most important MR instruments producer (Siemens [6]) is already trying to implement CS methods. More importantly, although it is quite new, this subject is very dynamically developing.

\section{References}

1. Bernstein, M. A., King, K. F., \& Zhou, X. J. (2004). Handbook of MRI pulse sequences. Elsevier.

2. Doneva, M., Mutapcic, A., \& Lustig, M. (2011). Compressed Sensing (CS) Workshop: Basic Elements of Compressed Sensing. Ohrid.

3. Foucart, S., \& Rauhut, H. (2013). A mathematical introduction to compressive sensing. Birkhauser.

4. Candès, E. J. (2008). The restricted isometry property and its implications for compressed sensing. C. $R$. Acad. Sci. Paris Ser. I, 346, 589-592. DOI: 10.1016/j. crma.2008.03.014.

5. Candès, E. J., \& Romberg, J. (2006). Robust uncertainty principles: Exact signal reconstruction from highly incomplete frequency information. IEEE Trans. Inform. Theor., 52(2), 489-509. DOI: 10.1109/ TIT.2005.862083.

6. Vincenti, G., Vincenti, G., Piccini, D., Monney, P., Chaptinel, J., Rutz, T., Coppo, S., Zenge, M. O., Schmidt, M., Nadar, M. S., Wang, Q., Chevre, P., Stuber, M., \& Schwitter, J. (2014). Preliminary experiences with compressed sensing MultiSlice cine acquisitions for the assessment of left ventricular function: CV sparse WIP. MAGNETOM Flash., 1, 18-26. 